\title{
Erratum to: Use of the Resection Map system as guidance during hepatectomy
}

\author{
Pablo Lamata - Félix Lamata - Valentin Sojar · Piotr Makowski • \\ Laurent Massoptier · Sergio Casciaro - Wajid Ali $\cdot$ Thomas Stïdeli • \\ Jérôme Declerck · Ole Jakob Elle • Bjørn Edwin
}

Published online: 17 April 2010

(C) Springer Science+Business Media, LLC 2010

\section{Erratum to: Surg Endosc}

Doi 10.1007/s00464-010-0915-3

The correct spelling of the ninth author's name is Ole Jakob Elle and the last author's name is Bjørn Edwin.

The correct full affiliations for Wajid Ali, Ole Jakob Elle and Bjørn Edwin are

The online version of the original article can be found under doi:10.1007/s00464-010-0915-3.

P. Lamata $\cdot$ J. Declerck

Siemens Molecular Imaging, Oxford, UK

F. Lamata

Hospital Clínico Universitario, Zaragoza, Spain

V. Sojar

University Clinik Center, Ljubljana, Slovenia

P. Makowski - L. Massoptier · S. Casciaro Institute of Clinical Physiology, Biomedical Engineering Division, National Council of Research (CNR-IFC),

73100 Lecce, Italy

W. Ali · O. J. Elle · B. Edwin

The Interventional Centre, Oslo University Hospital,

Oslo, Norway

W. Ali · O. J. Elle · B. Edwin

The Institute of Clinical Medicine, University of Oslo, Oslo, Norway

T. Stüdeli

Faculty of Industrial Design Engineering,

Delft University of Technology, Delft, The Netherlands

P. Lamata $(\square)$

Computing Laboratory, University of Oxford,

Wolfson Building, Parks Road, Oxford OX1 3QD, UK

e-mail: Pablo.lamata@hotmail.com
The Interventional Centre, Oslo University Hospital, Oslo, Norway and

The Institute of Clinical Medicine, University of Oslo, Oslo, Norway

The following collaborator was inadvertently omitted in the Acknowledgments:

We especially acknowledge A. M. Kazaryan for his help during data acquisition.

Delete the following in Reference 25: (for further reference): http://www.springerlink.com/content/j76732394677 $363 w / ? p=4693 b 05106 e f 4 b 109 c 37 b f 9 e 11 a e 0 f d 8 \& p i=0$ ) 